\title{
CROISSANCE DU FETUS DE VEAU DE GROSSESSE SIMPLE OU MULTIPLE A L'ÉPOQUE DE LA DIFFÉRENGIATION SEXUELLE (42 à 70 jours)
}

\author{
J. PRÉPIN, B. VIGIER et A. JOST \\ Laboratoire de Physiologie comparée, Université Paris VI, \\ 9, quai Saint-Bernard, \\ 75005 Paris \\ RÉSUMÉ,
}

Les variations en fonction de l'âge de la longueur du corps et du poids ont été analysées chez 215 fotus de Veau âgés de 42 à 70 jours, provenant soit de grossesses simples (un seul fœetus), soit de grossesses multiples (plusieurs fœetus).

Pendant cette période, il n'y a pas de différence de croissance significative pour $p=0,05$ ni entre les mâles et les femelles de gestation simple, ni entre les fœetus de gestation simple et ceux de gestation multiple.

Si l'on tient compte des intervalles de confiance (pour $p=0,05$ ) calculés pour les courbes de croissance, l'âge d'un fœetus de longueur ou de poids connu ne peut être estimé qu'à \pm 4 jours près. Cette approximation peut être insuffisante pour l'étude précise de certains processus de l'organogenèse sexuelle qui ont lieu pendant des phases successives relativement courtes.

\section{INTRODUCTION}

Au cours de recherches sur la différenciation sexuelle du foetus de Veau et sur le freemartinisme, nous avons collecté un nombre important de fœtus d'âge connu, entre 42 et 70 jours après l'insémination (JoST, VIGIER et PRÉPIN, I972). Une proportion importante de ces fœetus provenait de gestations multiples, produites en augmentant le nombre des ovulations par injection d'hormones gonado-stimulantes à la mère. Notre travail porte sur une période limitée du développement, quatre semaines sur une gestation de 280 jours environ.

Les points examinés sont les suivants : $\mathrm{I}^{0}$ existe-t-il une différence de croissance entre les fœtus mâles et femelles pendant la période considérée ; $2^{0}$ les foetus de ges- 
tations multiples suivent-ils la même courbe de croissance que ceux issus de grossesses ne comprenant qu'un seul fœetus (grossesses simples); $3^{\circ}$ dans les cas où des fœetus de même âge présentent des différences de taille importantes, le stade de différenciation de l'appareil génital est-il davantage en conformité avec l'âge ou avec la taille des fœtus? Nous n'avons pas étudié les relations éventuelles entre la croissance du fotus et celle des annexes embryonnaires (voir à ce sujet : TESTART et Du MESNIL Du Buisson, I966).

Dans la littérature, on ne trouve que des renseignements épars sur ces questions, en général sous la forme de remarques faites au sujet d'un petit nombre de foetus. MANEEIY (I952) a tracé une courbe de croissance du foetus de Veau entre I4 et 285 jours en utilisant les données antérieures de la littérature. WINTERS, GREEN et CoMsTOCK (I942) donnent de nombreux renseignements sur des fotus de 45 à 260 jours ; ils signalent en particulier que des fœtus de même poids peuvent avoir des âges différents et se trouver à des stades de développement différents. Selon WSETr, MATTHEwS et FoHRMAN (I948) la variabilité du poids des fœetus est importante jusqu'à 90 jours ; dans deux portées multiples, les fotus sont une fois plus lourds (à 237 jours) et une fois moins lourds (à ro2 jours) que ceux de gestations simples. Rowson et Dotr (1963) observent une grande variabilité dans la longueur du contour dorsal chez 20 fœtus de 37 jours. HAFEZ et RAJAkOSKI (I964) ont précisé la variabilité de la longueur et du poids des foetus de $30,45,60$ et 90 jours; selon eux, les fœtus de gestations multiples ont une taille et un poids normaux à 30 jours, mais légèrement réduits à 60 jours. D'autres auteurs ont étudié la croissance de divers organes en fonction de la longueur des fœetus (Nichols, I944), de leur poids (AвELoos, I946) ou de leur âge (GREEN, I946). Enfin GJESDAL (I969) a déterminé l'âge des foutus d'après le degré d'ossification du squelette, l'état des dents et du poil.

\section{MATÉRIEL, ET MÉTHODES}

\section{Animaux}

Sur les 215 fœtus étudiés, 203 sont de race Frisonne (père et mère), sept de race Normande quatre de race Charolaise et un de race Salers.

Les grossesses simples ont été obtenues par insémination naturelle ou artificielle de vaches gardées à la Station de Physiologie animale de l'I. N. R. A. à Jouy-en-Josas. Les vaches ont été sacrifiées un nombre déterminé de jours après l'insémination et l'utérus disséqué dans les dix minutes.

Les grossesses multiples ont été obtenues par super-ovulation expérimentale, en injectant 4 jours avant la date présumée des chaleurs 2 ooo à 3 ooo UI de PMSG par voie intramusculaire et en donnant le jour de l'insémination I 500 à 2 ooo UI d'HCG par voie intraveineuse (MAULEon, Benoit, Solari et Chupin, I970). Dans certaines portées multiples, des embryons morts avant le sacrifice ont été retrouvés macérés à côté des fotus vivants. lable).

Une seule grossesse multiple ( 2 fœtus) était spontanée (vache inséminée sans traitement préa-

\section{Mesure de la longueur et du poids des foetus}

Immédiatement après le prélèvement, les fœetus ont été placés en position naturelle sur du papier millimétré et mesurés du vertex à la racine de la queue. Les fotus de plus de 50 jours sont moins rigides que les plus jeunes, en particulier parce que leur tête devient mobile; pour les mesurer, on a placé la tête dans le prolongement du cớrs, nuque non pliée. 
Certains foetus ont été pesés après section du cordon ombilical au plus près de la paroi abdominale. Mais étant donné le gros diamètre du cordon ombilical, la manière dont il est coupé introduit une cause d'erreur; il en est de même pour la perte de sang plus ou moins importante subie par le fœtus.

\section{Détermination du sexe des foetus}

A partir de $4^{8}$ jours, et plus facilement après 52 jours, le sexe des fœetus peut être reconnu macroscopiquement d'après la distance ano-génitale. Les organes génitaux internes de tous les fœetus utilisés dans ce travail ont fait l'objet d'une étude histologique. Dans un certain nombre de cas, on a étudié les hétérochromosomes dans le foie fœtal (cf. Vraier, Prépin et Jost, I972).

\section{Méthodes statistiques}

La répartition des tailles ou des poids en fonction de l'âge est compatible avec une croissance de forme exponentielle (cf. fig. I) et l'évolution du logarithme de la longueur ou du poids des fœtus en fonction de l'âge peut être représentée par des droites (fig. 3). Les droites de régression $\mathrm{Y}=a+b \mathrm{X}$ ont été calculées suivant la méthode des moindres carrés (Simpson, RoE et LEwoNTIN, I960).

L'hypothèse d'une évolution linéaire de $\mathrm{Y}$ en fonction de l'âge se justifie par la répartition aléatoire des écarts des valeurs observées par rapport à la droite de régression.

Le coefficient de corrélation $r$ de Bravais-Pearson pour les différentes courbes de régression est compris entre 0,95 et 0,99 pour $(\mathrm{N}-2)$ degrés de liberté, ce qui indique qu'il y a une liaison très forte entre $\mathrm{X}$ et $\mathrm{Y}$ (cf. SIMPson et al., I960).

Pour comparer deux droites de régression, on vérifie si la différence entre deux coefficients de régression est significative en faisant un test en $t$ (cf. Simpson et al., I96o). Le $t$ calculé est comparé au $t$ théorique de Student pour $\mathrm{N}_{1}+\mathrm{N}_{2}-4$ degrés de liberté.

\section{Intervalle de confiance autour des droites de régression}

On a calculé les limites de confiance $\hat{\mathrm{Y}} \pm t \cdot \mathrm{S} \hat{\mathrm{Y}}$ des droites de régression pour les valeurs individuelles (cf. SNEDECOR, I965; pages I23, 125, 138) où :

Ŝ̀ $=$ écart type des déviations autour de la moyenne.

$\hat{\mathbf{Y}}=$ taille ou poids calculé à l'aide de l'équation de régression.

$t=t$ de Student correspondant à $(\mathrm{N}-2)$ degrés de liberté à une probabilité $p=0,05$.

L'intervalle de confiance est tel, qu'il n'y a que 5 p. roo de chances pour qu'un fœtus pris au hasard entre $4^{2}$ et $7^{\circ}$ jours soit en dehors de cet intervalle.

\section{RÉSULTATS}

\section{I. - Croissance des foetus de grossesses simples}

La longueur et le poids des fœetus mâles et femelles en fonction de leur âge sont rapportés dans les figures I et 2 . Entre 42 et 70 jours, la longueur du fotus est multipliée par 4 et le poids environ par 40.

L'aspect des deux courbes montre que s'il existe une différence de croissance entre les mâles et les femelles, elle ne peut être que très faible.

\section{Croissance en longueur.}

Sur la figure 3, sont portées en coordonnées semi-logarithmiques, les moyennes des longueurs des fotus de chaque classe d'âge, en fonction de l'âge, séparément 


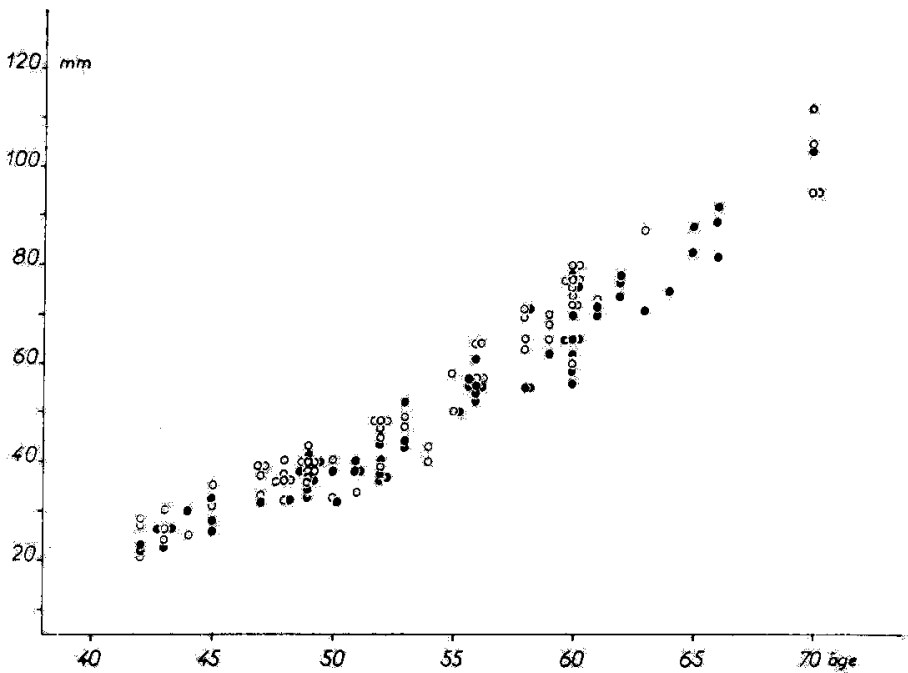

Fra. $\mathbf{x}$ - Longueur des fotus de grossesse simple en fonction de tráge $\circ$ foetus mâles;

- foetus femelles

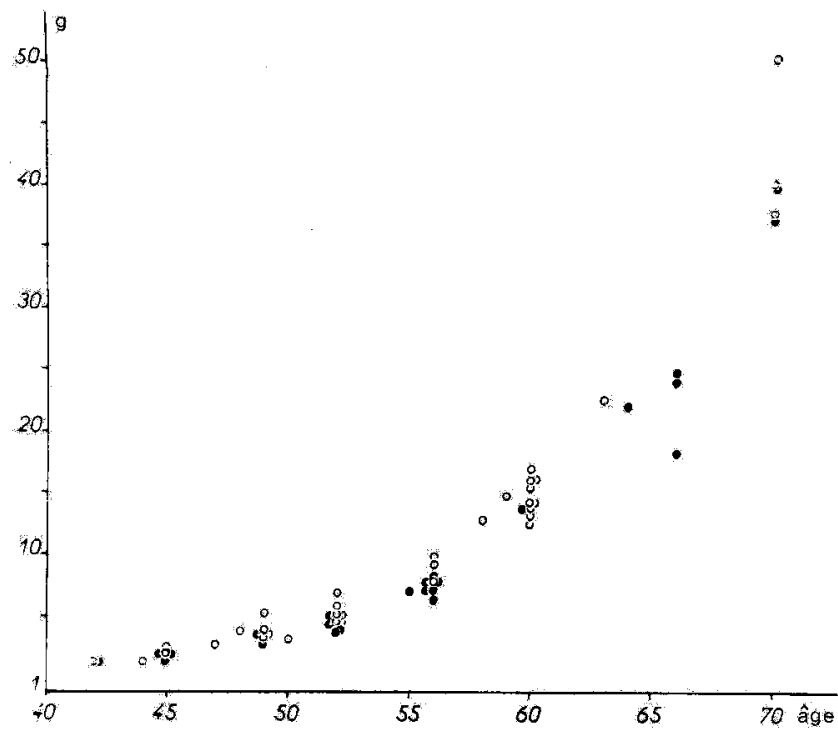

FIG. 2. - Poids des foetus de grossesse simple en fonction de l'age o foetus males;

- fotus ferielles 
pour les mâles et pour les femelles. Les deux courbes obtenues peuvent être assimilées à des droites d'équation :

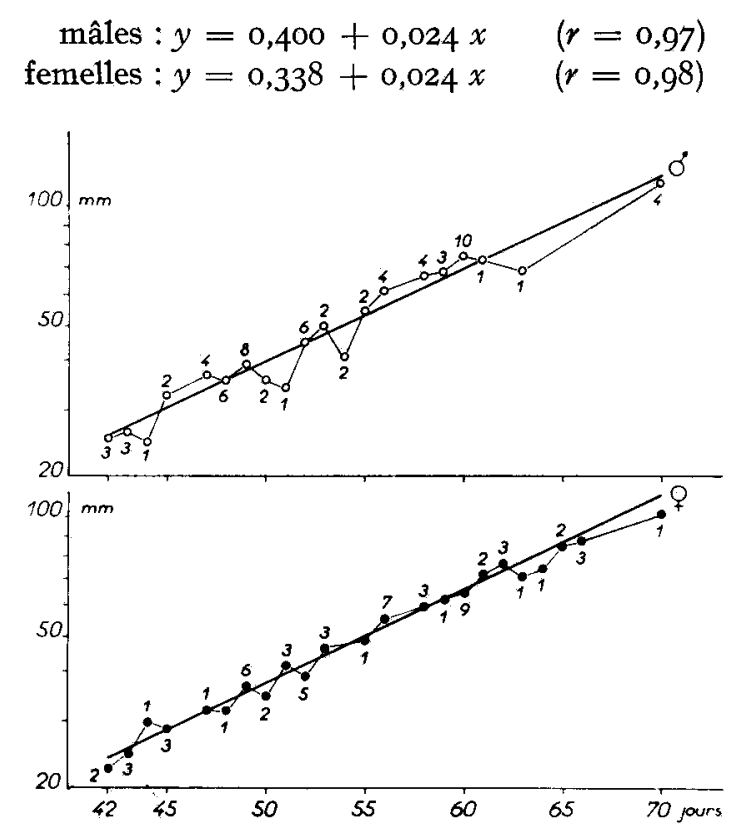

FIG. 3. - Evolution des tailles des fatus mâles (en haut) et des foetus femelles (en bas)

Chaque point représente la moyenne des longueurs des fœetus đu même âge (le chiffre indique leur nombre).

On a calculé la droite de régression correspondant aux valeurs individuelles. La distribution des valeurs moyennes est assez régulière autour des droites calculées. (mâles: $y=0,400+0,024 x$; femelles : $y=0,338+0,024 x)$.

Le test en $t$ indique que ces droites ne sont pas statistiquement différentes et qu'il n'y a donc pas de différence entre les mâles et les femelles pendant la période considérée. On peut donc utiliser une droite de régression commune pour les mâles et les femelles :

$$
y=0,380+0,024 x \quad(r=0,99)
$$

Les intervalles de confiance déterminés pour les valeurs individuelles (cf. Matériel et Méthodes) montrent que si on connaît la longueur d'un fœetus, ce renseignement seul ne permet de fixer son âge qu'à \pm 4 jours près (fig. 4 ).

Croissance pondérale.

Les données concernant les poids ont été traitées de la même manière et ont donné les mêmes résultats. Les équations des deux droites de régression sont très voisines et ne sont pas statistiquement différentes, ce qui indique qu'il n'y a pas de différence entre les mâles et les femelles :

$$
\begin{array}{rr}
\text { mâles : } y=0,052 x-\mathrm{r}, 995 & (r=0,97) \\
\text { femelles : } y=0,05 \mathrm{I} x-2,000 & (r=0,99)
\end{array}
$$

On peut utiliser une droite de régression commune pour les mâles et les femelles :

$$
y=0,052 x-2,000 \quad(r=0,98)
$$




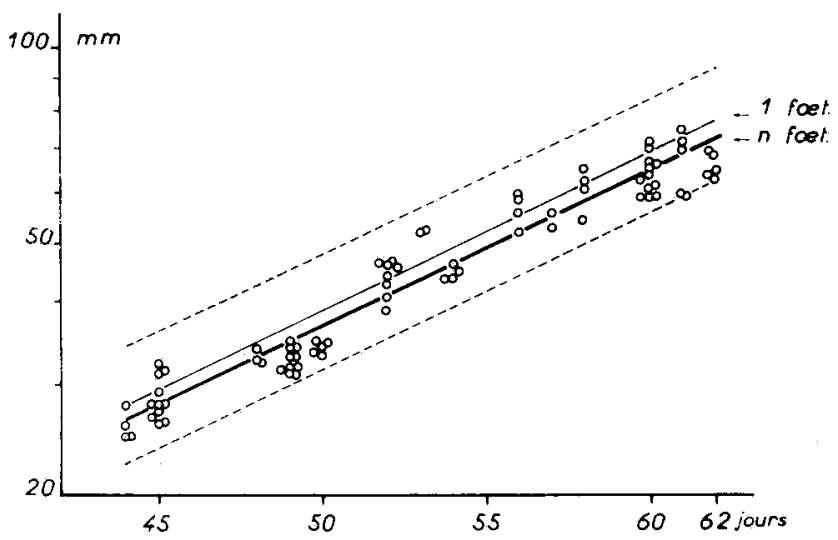

Fig. 4. - Longueur des fotus de grossesses multiples

placée par rapport à la droite de rêgression (I foet.)

calculée pour les foetus de grossesses simples âgés de 44 à 62 jours

$(y=0,024 x+0,356)$ et aux intervalles de confiance correspondants

La droite de régression ( $n$ fœt.), calculée pour les foetus de gestations multiples $(y=0,023 x+0,374)$ n'est pas statistiquement différente de la précédente. Les fotus de grossesses multiples sont placés à l'intérieur des intervalles de confiance calculés pour les fotus de grossesses simples.

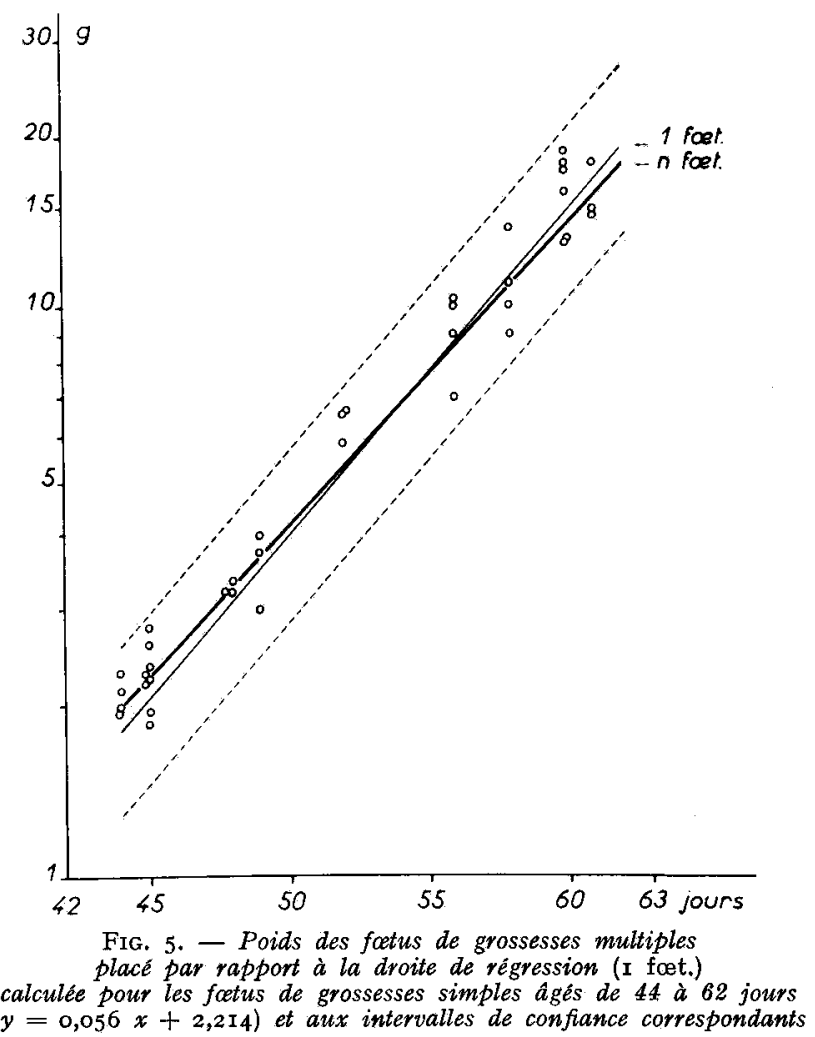

La droite de régression ( $n$ fot.), calculée pour les fœtus de gestations multiples $(y=0,054 x+2,073)$ n'est pas statistiquement différente de la précédente. Les fœetus de grossesses multiples sont placés à l'intérieur des intervalles de confiance calculés pour les foetus de grossesses simples. 
Les intervalles de confiance (fig. 5) montrent que si on connait, le poids d'un fœtus, on ne peut, à l'aide de ce renseignement, estimer son âge qu'à \pm 4 jours près.

\section{2. - Croissance des foetus de grossesses multiples}

Les longueurs et les poids des fœtus provenant de grossesses multiples sont rapportés dans le tableau 2. L'étude porte sur II portées de deux foetus, II portées de triplés, 6 portées de quađruplés et I portée de quintuplés.

\section{TABLEAU I}

Longueur et poids des fotus issus de gestation simple

La longueur et le poids correspondant à chaque fœtus sont indiqués dans le même ordre; les tirets correspondent aux fœetus qui n'ont pas été pesés

\begin{tabular}{|c|c|c|c|c|}
\hline \multirow{2}{*}{$\begin{array}{l}\text { Age } \\
\text { (jours) }\end{array}$} & \multicolumn{2}{|c|}{ Mâles } & \multicolumn{2}{|c|}{ Femelles } \\
\hline & Longueur (mm) & Poids (g) & Longueur (mm) & Poids (g) \\
\hline 42 & $21 ; 27 ; 28 ;$ & $-;-; 1,4 ;$ & $23: 22$; & $-\cdot 14 \cdot$ \\
\hline 43 & $26 ; 24 ; 30 ;$ & $-;-;-;$ & $26 ; 26 ; 23 ;$ & $-;-;-;$ \\
\hline 44 & 25 & 1,4 & $30 ;$ & \\
\hline 45 & $31 ; 35 ;$ & 2,$5 ; 2,3 ;$ & $26 ; 28 ; 32 ;$ & 1,$8 ; 2 ; 2,1 ;$ \\
\hline 47 & $33 ; 39 ; 39 ; 37 ;$ & 2,$8 ;-;-;-;$ & 32 & - ; \\
\hline 48 & $\begin{array}{l}40 ; 36 ; 37 ; 36 ; 36 ; \\
32 ;\end{array}$ & $\begin{array}{l}-;-;-;-; 3,9 ; \\
-;\end{array}$ & $32 ;$ & $-;$ \\
\hline 49 & $\begin{array}{l}40 ; 38 ; 36 ; 38 ; 40 \text {; } \\
37 ; 43 ; 40 ;\end{array}$ & $\begin{array}{l}-;-;-;-; 3,6 ; \\
5,4 ; 4 ; 3,7 ;\end{array}$ & $33 ; 41 ; 38 ; 37 ; 40 ; 34$ & 3,$3 ;-;-;-; 3,6 ;-$; \\
\hline $\begin{array}{l}50 \\
51\end{array}$ & $\begin{array}{l}40 ; 32,5 ; \\
34 ;\end{array}$ & $-; 3,3 ;$ & $\begin{array}{l}38 ; 32 ; \\
40 ; 38 ; 48 ;\end{array}$ & $\begin{array}{l}-;-; \\
-;-;-;\end{array}$ \\
\hline 52 & $\begin{array}{l}48 ; 48 ; 39 ; 47 ; 48 ; \\
45 ;\end{array}$ & $\begin{array}{l}5,4 ; 6 ; 4,8 ; 7,1 ; 5,0 ; \\
5,2 ;\end{array}$ & $44 ; 38 ; 37 ; 37 ; 40 ;$ & $4 ; 4,1 ; 5,2 ; 4,7 ;-;$ \\
\hline 53 & $49 ; 47 ;$ & $-;-;$ & $44 ; 43 ; 52 ;$ & $-;-;-$; \\
\hline 54 & $43 ; 40 ;$ & $-;-;$ & & \\
\hline 55 & $58 ; 50 ;$ & $-;-;$ & 50 & 7,$2 ;$ \\
\hline 56 & $57 ; 64 ; 64 ; 57$ & $-; 9,5 ; 10 ; 8 ;$ & $\begin{array}{l}56 ; 56 ; 57 ; 61 ; 56 ; \\
54 ; 53 ;\end{array}$ & $\begin{array}{l}8,2 ;-; 7,6 ; 8 ; 7,5 ; \\
8 ; 6,7 ;\end{array}$ \\
\hline 58 & $70 ; 71 ; 63 ; 65 ;$ & $-;-;-; 13 ;$ & $55 ; 55 ; 71 ;$ & $-;-;-;$ \\
\hline 59 & $70 ; 68 ; 65 ;$ & $-; 15 ;-;$ & 62 & - ; \\
\hline 60 & $\begin{array}{l}80 ; 77 ; 74 ; 80 ; 72 ; \\
76 ; 77 ; 72 ; 77 ; 60 \text {. }\end{array}$ & 16,$3 ; 17,2 ; 14 ; 16 ; 13,9 ;$ & $\begin{array}{l}56 ; 59 ; 65 ; 65 ; 76 ; \\
65 ; 78 ; 70: 62:\end{array}$ & $-;-;-;-; 14 ;$ \\
\hline 61 & $73 ;$ & $\begin{array}{l}0,4,14,7,10,17,4,-1 \\
-;\end{array}$ & $\begin{array}{l}70 ; 72 ; \\
70 ; 02 ;\end{array}$ & $\begin{array}{l}-;-;-;-; \\
-;-;\end{array}$ \\
\hline 62 & & & $74 ; 78 ; 77 ;$ & $-;-;-;$ \\
\hline 63 & 87 ; & 22,8 & $71 ;$ & - ; \\
\hline 64 & & & 75 & 22,3 \\
\hline 65 & & & $88 ; 83 ;$ & $-;-;$ \\
\hline 66 & & & $92 ; 89 ; 82 ;$ & 24,$9 ; 24,2 ; 18,5 ;$ \\
\hline 70 & $95 ; 95 ; 112 ; 105 ;$ & $38 ; 40,5 ; 50,6 ; 40,3 ;$ & 104 & 37,6 \\
\hline
\end{tabular}

La comparaison des tableaux I et 2 montre qu'il n'y a pas de différence constante entre les fœtus de grossesses simples ou multiples. Dans 1'ensemble la variabilité est du même ordre pour chaque stade ; cependant, à 49 jours, plusieurs des fœetus degros- 
sesses doubles, triples ou quadruples ont une taille à peine égale aux plus petits des fœetus du même âge issus de grossesse simple. Les triplés de $6 \mathrm{r}$ jours ont des mensurations normales alors que ceux de 62 jours sont un peu petits. Les quadruplés de 54 jours ou les quintuplés de 45 jours sont très bien développés.

Pour l'étude statistique, tous les fœtus de grossesses multiples, ont été groupés quel que soit leur nombre par portée, car à l'intérieur de chaque catégorie (jumeaux,

TABLEAU 2

Longueur et poids des fretus issus de gestations multiples

Chaque ligne représente une portée;

les tirets correspondent aux fœetus qui n'ont pas été pesés

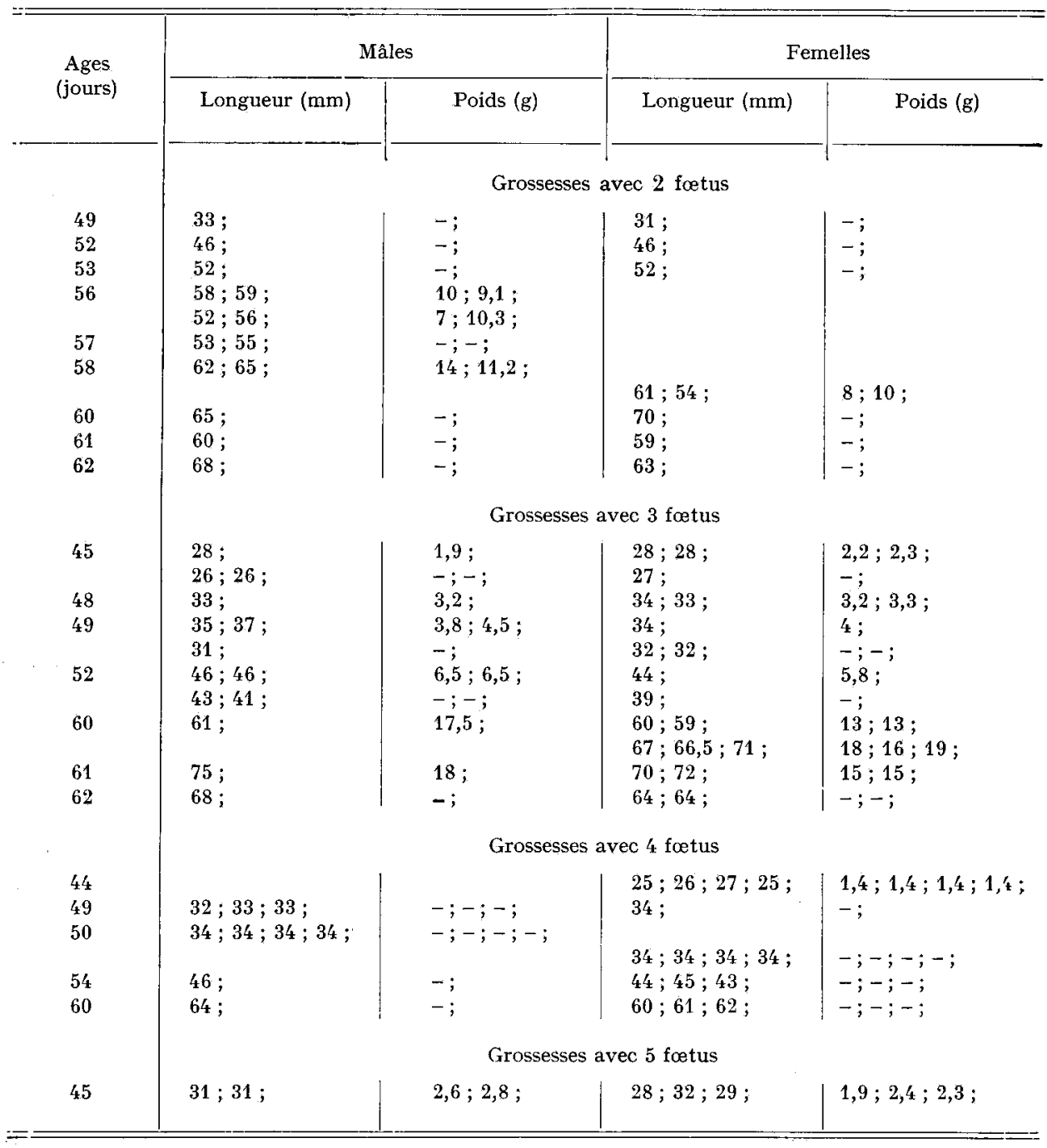


triplés, etc.) le nombre de fotus aurait été trop peu nombreux pour être traité séparément. On a analysé la croissance de ces fœtus, de deux manières : d'une part en étudiant la répartition de leur longueur ou de leur poids par rapport à l'intervalle de confiance calculé pour les fœtus de grossesse simple des mêmes âges ( 44 à 62 jours) (fig. 4 et 5) et d'autre part en calculant les droites de régression de leurs longueurs ou de leurs poids en fonction de l'âge.

\section{Croissance en longueur.}

Tous les fœtus de grossesse multiple se placent dans l'intervalle de confiance calculé pour les foetus de grossesse simple (fig. 4), mais la répartition n'est pas homogène : $5^{8}$ points se trouvent en dessous de la droite de régression, I4 seulement au dessus et 7 sur la droite. La droite de régression de la longueur des fœetus de gestations multiples $(y=0,374+0,023 x)$ se situe légèrement en dessous de celle correspondant aux foetus de gestation simple âgés de 44 à 62 jours $(y=0,356+0,024 x)$. Mais le test en $t$ de Student montre que les deux droites ne sont pas significativement différentes pour $p=0,05$.

Ainsi, bien que les foetus de grossesse multiple aient tendance à être un peu moins longs que les fœtus de grossesse simple, cette différence n'est pas statistiquement significative.

\section{Croissance pondérale.}

Plusieurs foetus n'ont pas été pesés ; l'étude porte donc sur un nombre de fotus moins élevé que précédemment.

Les poids des fottus de grossesses multiples âgés de 44 à 62 jours se placent d'une manière relativement homogène dans l'intervalle de confiance calculé pour les foetus de gestation simple de même âge (fig. 5). Cependant les poids des plus jeunes foetus de grossesse multiple se trouvent dans leur grande majorité au-dessus de la droite de régression déterminée pour les fotus de gestation simple.

La droite de régression du poids en fonction de l'âge, calculée pour les foetus de gestation simple $(y=0,054 x-2,073)$ rend compte de ce fait, bien qu'elle ne soit pas statistiquement différente de celle calculée pour les foetus de grossesse simple âgés de 44 à 62 jours $(y=0,056 x-2,2$ I 4$)$.

Pour le lot de fotus étudiés entre 44 et 62 jours, il n'apparaît pas de différence nette du poids par rapport aux fotus de grossesse simple.

\section{3. - Variations dans l'état de l'appareil génital en fonction de l'áge ou de la taille}

Beaucoup de recherches sur l'organogenèse sexuelle du Veau ont été faites sur des foetus collectés aux abattoirs donc d'âge inconnu. Or deux foetus de même longueur peuvent, en fait, avoir une différence d'âge de près d'une semaine. Il est donc important de préciser si 1'organogenèse sexuelle est en relation surtout avec l'âge du foetus ou avec sa taille. Nous examinerons deux caractères sexuels se différenciant pendant la période étudiée et pour lesquels nous avons suffisamment de données numériques. 
a) Distance ano-génitale.

Chez les fotus mâles, à partir de 47 jours, le pénis migre ventralement en direction de l'ombilic qu'il atteint à 58 jours (Jost et al., I972); la distance entre l'anus et l'orifice uro-génital s'accroît donc rapidement chez le mâle en fonction de l'âge, mais non chez la femelle (fig. $6 \mathrm{~A}$ ). La même conclusion apparaît si l'on représente les
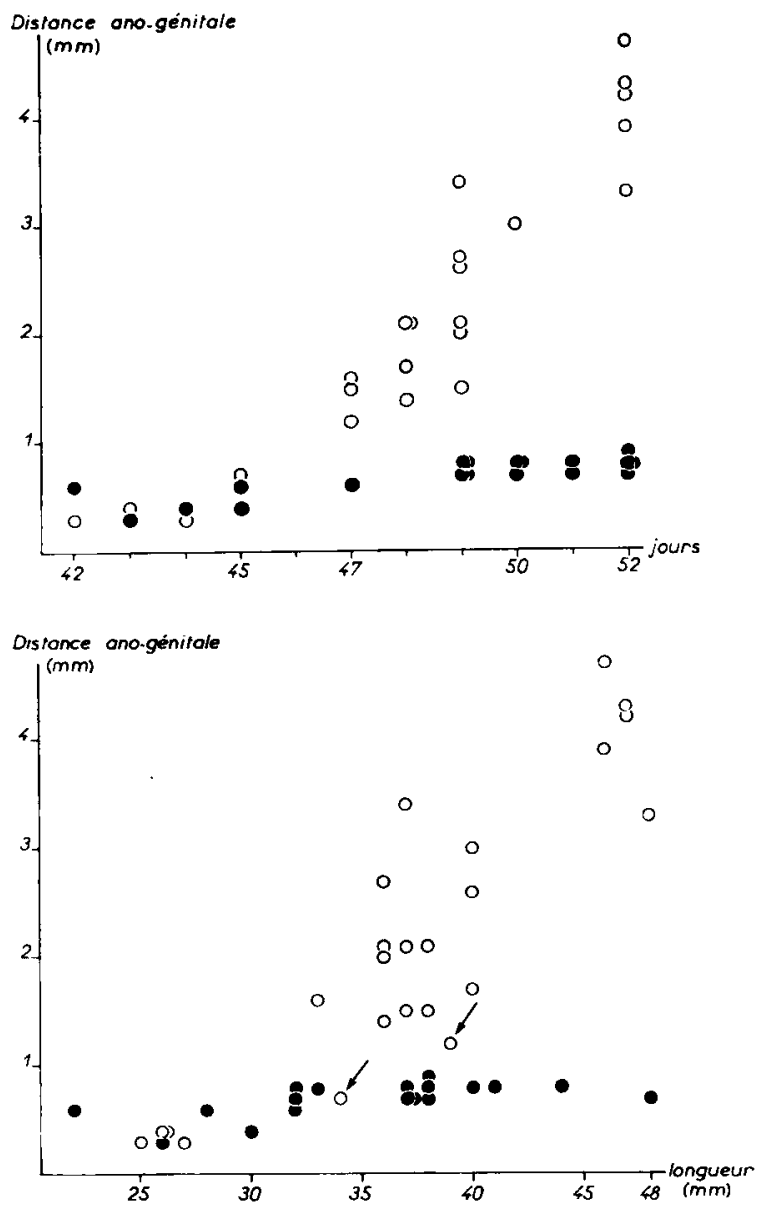

FIG. 6. - Distances ano-génitales des foetus de Veau mâles (0) et femelles (๑), soit en fonction de l'âge (en haut), soit en fonction du poids du foetus (en bas)

Noter les deux points indiqués par les flêches

distances ano-génitales en fonction de la longueur des mêmes fotus (fig. 6 B) ; cependant dans ce dernier graphique deux fotus ont une distance ano-génitale courte par rapport à d'autres fœitus de même taille : il s'agit de foetus particulièrement grands pour leur âge (fœtus de 45 jours mesurant $34 \mathrm{~mm}$, alors que la moyenne pour cet âge est de $28,8 \mathrm{~mm}$ d'après la courbe de régression ; fotus de 47 jours mesurant $39 \mathrm{~mm}$ au lieu de la taille moyenne calculée de $32,2 \mathrm{~mm}$ ). Ces deux foetus ont un âge qui cor- 
respond au début de la période critique durant laquelle la distance ano-génitale des mâles s'accroît brusquement (47-49 jours). A ce moment, 1a distance ano-génitale dépend donc plus de l'âge du fœetus que de sa taille.

\section{b) Longueur de l'urètre supérieur non sexuel.}

Avant le début de la différenciation sexuelle du fotus, les canaux de Wolff (qui deviendront les canaux déférents du mâle) débouchent dans le sinus urogénital à une faible distance sous le futur col de la vessie. Chez le mâle, ce carrefour reste situé assez près de la vessie et la partie antérieure non sexuelle de l'urètre reste courte ; la partie postérieure de l'urètre, située en arrière de ce niveau servira à la fois de canal génital et de canal urinaire. Au contraire, chez la femelle, ce carrefour se déplace vers la partie postérieure du corps au fur et à mesure que se développe le vagin, et l'urètre purement urinaire s'allonge beaucoup après 55 jours. Cet allongement est fonction de l'âge, comme cela se voit si l'on compare des foetus de même taille mais d'âge différent (tabl. 3). Mais pour un même âge, il semble y avoir une relation entre

TABLEAU 3

Longueur de l'urètre antérieur non sexuel

(entre le col de la vessie et l'abouchement des canaux de Wolff) chez des fotus femelles de Veau

\begin{tabular}{|c|c|c|c|}
\hline \multirow{2}{*}{$\underset{\text { (jours) }}{\text { Age }}$} & \multicolumn{2}{|c|}{ Longueur } & \multirow{2}{*}{$\begin{array}{l}\text { Rapport } \\
\text { uretre } \\
\text { fotus }\end{array}$} \\
\hline & $\begin{array}{c}\text { Fœtus } \\
\text { (mm) }\end{array}$ & $\begin{array}{l}\text { Urètre } \\
(\mathrm{mm})\end{array}$ & \\
\hline 55 & $\begin{array}{l}32 \\
50\end{array}$ & $\begin{array}{l}0,35 \\
0,50\end{array}$ & $\begin{array}{l}1,09 \\
1,96\end{array}$ \\
\hline 56 & $\begin{array}{l}\mathbf{5 6} \\
\mathbf{5 6} \\
\mathbf{5 7} \\
\mathbf{6 1}\end{array}$ & $\begin{array}{l}1,10 \\
1,12 \\
0,95 \\
1,10\end{array}$ & $\begin{array}{l}1,96 \\
2,00 \\
1,66 \\
1,80\end{array}$ \\
\hline 58 & 56 & 1,25 & 2,23 \\
\hline 59 & 59 & $1, \mathbf{4 0}$ & 2,37 \\
\hline 60 & $\begin{array}{l}60 \\
65 \\
71 \\
78\end{array}$ & $\begin{array}{l}1,40 \\
1,50 \\
1,87 \\
1,75\end{array}$ & $\begin{array}{l}2,33 \\
2,30 \\
2,64 \\
2,24\end{array}$ \\
\hline 64 & 75 & 2,75 & 3,66 \\
\hline 66 & 92 & 3,37 & 3,66 \\
\hline 70 & 104 & 3,25 & 3,12 \\
\hline
\end{tabular}

la longueur de l'urètre et la longueur du corps (comparer les divers fotus de 59 et 60 jours). Ce caractère sexuel dépend donc à la fois de l'âge du fotus et, pour un âge donné, de sa taille. 


\section{DISCUSSION}

Les longueurs et les poids donnés par WinTERS et al. et par MANNELy pour les fœetus âgés de 45 à 70 jours sont dans l'ensemble en accord avec nos mesures : les 5 fotus étudiés par Winters et al. et I4 des 17 fœetus rapportés par Mannel, y se trouvent à l'intérieur des intervalles de confiance calculés sur notre matériel. Les trois cas qui font exception dans les données de ManNELY ont été empruntés au travail antérieur de BERGMANN (I925).

Les différences sexuelles en ce qui concerne la croissance fœetale ont été relativement peu étudiées. Dans l'espèce humaine les foetus masculins sont plus grands et plus lourds que les foetus féminins dès $1 \mathrm{a} 24^{\mathrm{e}}$ semaine de la gestation, les stades antérieurs n'ayant pas été étudiés (LUBCHENKo et al., I963). Chez le Veau, une différence semblable a été signalée (KEILLER, I920 ; BEER, I925 ; SWETT, MATTHEws et FoHRMAN, r948), mais nous n'avons pas trouvé de travail détaillé sur ce sujet. Dans la population étudiée ici, entre 42 et 70 jours de vie fœtale, il n'y a pas de différence de taille entre les deux sexes; il est probable qu'une différence apparaît à une date plus tardive, puisqu'à la naissance, le poids des veaux mâles est supérieur à celui des femelles (Fitch, Mc GiLIIARD et DRUM, I924; MEYER, I964).

Le nombre de foetus par portée est un autre facteur retentissant sur le poids et la taille des jeunes à la naissance (cf. DAwES, I968). Dans l'espèce humaine, le nombre de foetus n'influence leur poids individuel qu'après la $26^{\text {e }}$ semaine (cf. WIDDowson, I968). Chez le Veau, HAFEz et RaJAkoski (1964), notent que les foetus de grossesse multiple sont, dès 60 jours, plus petits que ceux provenant de grossesse unique. D'après TAntawy et Ahmed (I957), et Donald (I958), les foetus issus de gestation multiple sont plus petits à la naissance que ceux provenant de gestation simple.

L'étude faite ici, sur 79 fœtus de grossesses multiples, âgés de 45 à 62 jours, ne fait pas apparaître de différences significatives entre les fotus de grossesses simples et ceux de grossesses multiples, bien que vers la fin de la période considérée les foetus de gestations multiples aient tendance à être plus petits que ceux de gestation simple. Il est donc probable que les différences apparaissent surtout après les deux premiers mois de gestation.

La majorité des travaux concernant l'organogenèse sexuelle chez le foetus de Veau et chez les freemartins, a été faite sur des embryons prélevés aux abattoirs, donc d'âge conceptionnel non connu. Or les événements importants de l'organogenèse sexuelle se déroulent suivant une chronologie précise, pendant des phases successives relativement courtes. Puisque deux fœtus de même taille peuvent avoir une différence d'âge de 8 jours il est difficile de fonder une étude chronologique seulement sur les tailles respectives des fœus, à moins de disposer d'une assez grand nombre de cas. La définition de l'âge physiologique du fœutus pose en effet des problèmes complexes qui ont été discutés antérieurement (cf. JosT, r954 ; Jos'T et PICON, I970). L'intensité de certaines fonctions physiologiques augmente brusquement à un stade donné : les variations individuelles d'un fœetus à l'autre sont spécialement grandes au stade où survient ce changement, alors qu'elles sont moindres soit avant, soit après ce stade. 
Pour beaucoup de paramètres, l'âge du foetus est un critère important, mais le poids atteint à un âge donné joue un rôle également (cf. JOST, MOREAU et FouRNIER, I960). L'idéal pour les travaux consacrés à la physiologie du développement est de connaître non seulement l'âge des fotus, mais aussi leur taille et leur poids ainsi que la variabilité caractérisant chacun des stades étudiés.

Reçu pour publication en mai 1972.

\section{REMERCIEMENTS}

Ce travail, réalisé dans le cadre d'un contrat avec la D. G. R. S. T, portant sur le freemartinisme, a été rendu possible grâce à l'aide des membres de la Station de Physiologie animale de l'I. N. R. A. à Jouy-en-Josas. Nous remercions en particulier le professeur Ch. ThIBAULT, MM. R. Ortavant, P. Maulíon et F. du Mesnil du Buisson et Mme M. Gerard pour leur précieuse collaboration; Mlle A. SolARI, ingénieur à l'I. N. R. A., qui nous a conseillés dans l'élaboration de l'analyse statistique; MM. Y. de Fontaubert et F. de La Chevalerie, qui ont traité les animaux en expérience.

\section{SUMMARY}

GROWTH OF THE CALF FETUS OF SINGLE OR MULTIPLE PREGNANCIES DURING THE PERIOD OF SEXUAL, DIFFERENTIATION (42-70 DAYS)

Variations according to age, body length, and weight were analyzed in 2 I 5 calf fetuses, 42-7o days old. These fetuses came from single (only one fetus) or multiple (several fetuses) pregnancies.

Ditring this period, there was no significant difference in growth ( $p=0.05)$ either between males and females of single pregnancies or between fetuses of single and of multiple pregnancies.

The age of a fetus of known length and weight can only be determined with an incertitude of \pm 4 days, taking into account the confidence intervals $(p=0.05)$ calculated for the growth curves.

This approximation may not be sufficient for a detailed study of some sexual development processes which occur during relatively short successive phases.

\section{RÉFÉRENCES BIBLIOGRAPHIQUES}

Abeloos M., 1946. Phases et étapes de la croissance fotale du Veau. C. R. Acad. Sci. Paris, 222, 24I242.

Beer G., 1925. Jahresber. Vet. Med. (abstr.), 45, 237. Cité par E. B. Harvey (I959).

Dawes G. S., I968. Foetal and Neonatal Physiology. Year Book Medical Publishers, Inc., Chicago.

Donald H. P., I958. Proc. 10th Int. Conf. Genet., 1, 225. Cité par G. C. Everitt in "Growth and development of Mammals ", edited by G. A. Lodge and G. E. Lamming, p. 133, 1968, Butterworth, London.

Fitch J. B., Mc Gililiard P. C., Drum G. M., I924. J. Dairy Sci., 7, 222. Cité par E. B. Harvey (1959).

Gjesdal F., 1969. Age determination of bovine fcetuses. Acta vet. scand., 10, 197-218.

GrenN W. W., I946. Comparative Growth of the Sheep and Bovine animal during prenatal life. Am. J. Vet. Res., 7, 395-402.

Hafez E. S. E., Rajakoski E., I964. Placental and fetal development during multiple bovine pregnancy. Anatomical and physiological studies. Anat. Rec., 150, 303-3I6. 
HaRvey E. B., I959. Implantation, Development of the Fetus, and Fetal Membranes, in Reproduction in Domestic animals, edited by H. H. Cole and P. T. Cupps. Academic Press, N. Y., vol. I ; 433-468, 1959.

Jost A., I954. Hormonal factors in the development of the fetus. Cold Spring Harbor Symp. Quant. Biol., 19, 167-r80.

Jost A., Moreau G., Fournier C., ig6o. Date et ordre d'apparition des premiers centres d'ossification chez le foetus de rat normal ou soumis au propylthiouracile. Arch. Anat. microsc. Morphol. experim., 49, 43I-457.

Jost A., Picon L., I97o. Hormonal control of fetal development and metabolism. Adv. metab. disorders, 4, 123-184.

Jost A., Vigier B., Prépin J., I972. Freemartins in cattle : the first steps of sexual organogenesis. J. Reprod. Fert., 29, 349-379.

Keller K., I920. Wiener. Tierärztl. Monatsschr., 7. I937. Cité par E. B. Harvey (1959).

Lubchenco L. O., Hansman C., Dressler M., Boyd E., I963. Intrauterine growth as estimated from liveborn birth weight data at 24 to 42 weeks of gestation. Pediatrics, 32, 793-800.

Maneely R. B., 1952. Note on the ageing of Bovine embryos. Vet. Rec., 35, 509-5II.

Mauléon P., Mariana J.-C., Benoit M., Solari A., Chipin D., r97o. Influence de différentes doses de PMS et HCG, injectées en phase folliculaire du cycle œstrien, sur le nombre et le rendement d'ovulations de vaches de race Franfaise Frisonne pie-noire. Ann. Biol. Anim. Bioch. Biophys., 10, horssérie, $3 \mathbf{r}-46$.

MEYeR H., I964. Züchtungskunde, 36 ; 299. Cité par ST. C. S. TAYLOR in Growth and development of Mammals, edited by G. A. Lodge and G. E. Lamming, p. 272, I968, Butterworth, London.

Nichols C. W., I944. The embryology of the calf : Fetal growth weights, relative age and certain body measurements. Am. J. Vet. Res., 5, 135-141.

Rowson L. E. A., Dotт H. M., I963. A Hazard of pregnancy diagnosis in cattle : early foetal size. Vet. Rec., 75, 865-866.

Simpson G. G., Roe A., Lewontin R. C., rg6o. Quantitative Zoology. Harcourt, Brace and Company, Inc., r96o. N. Y., Birlingame.

SNedecor G. W., 1956. Statistical Methods. 5th ed. The Iowa State University Press.

Swett W. W., Matrews C. A., Fohrman M. H., 1948. Development of the foetus in the dairy cow. Technical Bulletin, 964, 34 pages.

Tantawy A. O., Ahmed I. A., 1957. Emp. J. exp. Agric., 25, 24. Cité par E. S. E. HAfez, M. R. JAINUDEEN et D. R. LINDSAY, 1965. Gonadotropin-induced twinning and related phenomena in beef cattle. Acta Endocr., suppl. I02.

Testart J., du Mesnil du Buisson F., ig66. Étude biométrique des placentomes dans les gestations simples ou gémellaires des Bovins. Ann. Biol. anim. Bioch. Biophys., 6, 483-493.

Vigier B., Prépin J., Jost A., 1972. Absence de corrélation entre le chimérisme XX/XY dans le foie et les premiers signes du freemartinisme chez le foetus de Veau. Cytogenetics, 11, 8r-Ior.

Widdowson E. M., 1968. Growth and composition of the fetus and newborn, in Biology of gestation r-49, vol. 2, edited by N. S. Assali. Academic Press. N. Y., London.

Winters L. M., Green W. W., Comstock R. E., 1942. Prenatal development of the Bovine. Minn. Agr. Exp. Sta. Techn. Bull., 151, 50 pages. 